\title{
Clustering Algorithms for Huge Datasets: A Mathematical Approach
}

\author{
Shyam Mohan J. S. \\ Assistant Professor, Dept. Of CSE, SCSVMV, \\ Enathur, Kanchipuram, Tamilnadu, India
}

\author{
Shanmugapriya $\mathrm{P}$. \\ Associate Professor, Dept. Of CSE, SCSVMV, \\ Enathur, Kanchipuram, Tamilnadu, India
}

\begin{abstract}
Identifying clusters for huge datasets are useful for finding out attributes of a particular dataset and thereby providing insights for making effective decision making. In our previous work, we have proved the concept of clustering algorithms for huge datasets theoretically by applying small computations on the available datasets. In this paper, we extend the same work by applying Mathematical calculations for the datasets so as to prove the correctness of our previous work carried out. Our proposed method is applied to various datasets and proved KMeans algorithm mathematically and the experimental calculations performed on various clustering algorithms shows that our approach provides the new idea of clustering techniques that can be applied for any number of huge and complex datasets.
\end{abstract}

\section{Keywords}

Machine Intelligence, Clustering Algorithms

\section{INTRODUCTION}

Classifying dataset into groups can be effectively done by Clustering where data points in a particular group share similar features. [1] Some of the applications where Clustering is widely used are: pattern recognition, Customer segmentation, stock market clustering, reduced dimensionality for effective data mining etc.Cluster analysis is done in many ways like $\mathrm{K}$ means, fuzzy means etc.[2][3]

Majority of clustering algorithms fail because of the total number of iterations performed over datasets grows exponentially in size. Big data refers to datasets of huge size. Batch processing or parallel programming technique (MapReduce) provides effective processing of huge datasets. MapReduce is easily scalable that runs on any hardware. The concept of MapReduce is already discussed in our previous works and papers.[4] Parallel programming using MapReduce reduces the time complexity for processing cluster analysis.[5].

\section{BASICS OF DISTANCE AND}

\section{SIMILIARITY CALCULATION}

For effective clustering, distance (dissimilarity) and similarity measures form the basic idea.[6]. For quantitative datasets, distance is used to find the relationship among data and even similarity features are used for qualitative data.

\subsection{Distance Functions}

\section{Minkowski distance}

For a normal vector space, the Minkowski distance is used between two points.

$$
d(x, y)=\left(\sum_{i=0}^{n-1}\left|x_{i}-y_{i}\right|^{p}\right)^{1 / p}
$$

Special cases:

- When $\mathrm{p}=1$, It is known as the Manhattan distance.

- When $\mathrm{p}=2$, It is known as the Euclidean distance.

- In the limit that p --> +infinity, the distance is known as the Chebyshev distance.

\section{Euclidean distance}

For a Euclidean space, distance between two is known as Euclidean distance.

$$
d(x, y)=\left(\sum_{i=0}^{n-1}\left|x_{i}-y_{i}\right|^{2}\right)^{2}
$$

Manhattan distance

$\mathrm{d}(\mathrm{x}, \mathrm{y})=\sum_{i=0}^{n-1}\left|x_{i}-y_{i}\right|$

where $\mathrm{x}$ and $\mathrm{y}$ represent two vectors of length $\mathrm{n}$.

\section{Chebyshev distance}

In any vector space, the greatest difference between any coordinate direction is the distance between two vectors. $d(x, y)=\lim _{n \rightarrow \infty}\left(\sum_{i=0}^{n-1}\left|x_{i}-y_{i}\right|^{p}\right)^{1 / p}=$ $\max _{i=0--------n-1}\left|x_{i}-y_{i}\right|$

\section{Pearson correlation distance}

The correlation distance between two sample vectors in a Pearson's product-momentum is called Pearson correlation distance. The value of correlation coefficient is between $[-1,1]$, the Pearson distance lies in $[0,2]$ and measures the linear relationship between the two vectors.

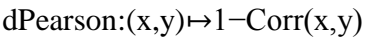

Spearman correlation distance

The spearman correlation method is used to compute the correlation between the rank of $\mathrm{x}$ and the rank of $\mathrm{y}$ variables where $\mathrm{x}$ and $\mathrm{y}$ sequences are ranked separately. At each position $\mathrm{i}$, the differences in rank are calculated. The distance between sequences $\mathrm{X}=(\mathrm{X} 1, \mathrm{X} 2, \ldots \ldots)$ and $\mathrm{Y}=(\mathrm{Y} 1, \mathrm{Y} 2, \ldots)$ is computed using the following formula:

$$
1-6 \sum_{\mathrm{i}=1}^{\mathrm{n}} \frac{\left(\operatorname{rank}\left(\mathrm{x}_{\mathrm{i}}\right)-\operatorname{rank}\left(\mathrm{y}_{\mathrm{i}}\right)\right)^{2}}{\mathrm{n}\left(\mathrm{n}^{2}-1\right)}
$$

$\boldsymbol{X} i$ and $\boldsymbol{Y} i$ represent the $i$ th values of $\boldsymbol{X}$ and $\boldsymbol{Y}$.

In general the value of Spearman Correlation in the range of 1 to 1 .

Kendall correlation distance

$$
\mathrm{K}\left(T_{1}, T_{2}\right)=\sum_{\{i, j\} \in P} K_{i, j}\left(T_{1}, T_{2}\right)
$$


Where $P$ is the set of unordered pairs of distinct elements in $T_{1}$ and $T_{2}$

$K_{i, j}\left(T_{1}, T_{2}\right)=0$ if $i$ and $j$ are in the same order in $T_{1}$ and $T_{2}$ $K_{i, j}\left(T_{1}, T_{2}\right)$

$=1$ if $i$ and $j$ are in the opposite order in $T_{1}$ and $T_{2}$

\section{STANDARD K-MEANS}

The standard K-means algorithm is an iterative process that guarantees a decrease in total error (value of the objective function $\mathrm{f}(\mathrm{M})$ ) on each step [7][8].The algorithm is as follows:

1. Choose $\mathrm{k}$ initial means s1,s2,---------sn , uniformly at random from the set $\mathrm{X}$.

2. For each point $\mathrm{x} \in \mathrm{X}$, find the closest mean si and add $\mathrm{x}$ to a set Ai.

3. For $\mathrm{i}=1,2,----\mathrm{k}$, set $\mathrm{si}$ to be the centroid of the points in Ai.

4. Repeat steps 2 and 3.

5. Step 4 is reached when the optimal solution is obtained. The algorithm takes $\mathrm{O}(\mathrm{nkd})$ time for execution.

\section{COMPUTING K-MEANS IN R}

Computing $\mathrm{K}$-means in $\mathrm{R}$ is done by calculating $\mathrm{K}$-means. This is done by grouping datasets into clusters viz, centers $=2$, and thereby clusters of 2 .We can set the K-means function to start and stop.

Example:

We take two objects A and B with the values tabulated as follows:

Table I : Objects A and B with their coordinate points

\begin{tabular}{|c|c|c|c|c|c|c|}
\hline & $\begin{array}{c}\text { values } \\
\text { at } \mathbf{1}\end{array}$ & $\begin{array}{c}\text { Values } \\
\text { at 2 }\end{array}$ & $\begin{array}{c}\text { Values } \\
\text { at } \mathbf{3}\end{array}$ & $\begin{array}{c}\text { Values } \\
\text { at } \mathbf{4}\end{array}$ & $\begin{array}{c}\text { Values } \\
\text { at 5 }\end{array}$ & $\begin{array}{c}\text { values } \\
\text { at 6 }\end{array}$ \\
\hline $\begin{array}{c}\text { Object } \\
\text { A }\end{array}$ & 18 & 20 & 30 & 21 & 34 & 32 \\
\hline $\begin{array}{c}\text { Object } \\
\text { B }\end{array}$ & 100 & 200 & 150 & 300 & 350 & 450 \\
\hline
\end{tabular}

Minkowski distance

For input value $\varphi=4$,

Minkowski distance $=467.7$

Euclidean distance

Object $A=\{18,20,30,21,34,32\}$

Object $B=\{100,200,150,300,350,450\}$

$(x, y)=\{(18,100),(20,200),(30,150),(21,300),(34,350),(32,450)\}$

Euclidean distance between $(18,100)$ and $(32,450)$ :

$\mathrm{d}=350.279888$
Table II : K-Means Calculation

\begin{tabular}{|cc|}
\hline age & spend \\
\hline Min. :18.00 & Min. : 100.0 \\
1st Qu.:20.25 & 1st Qu.:162.5 \\
Median :25.50 & Median :250.0 \\
Mean :25.83 & Mean :258.3 \\
3rd Qu.:31.50 & 3rd Qu.:337.5 \\
Max. :34.00 & Max. :450.0 \\
\hline
\end{tabular}

Chebyshev distance

Table III : Chebyshev distance Calculation

\begin{tabular}{|l|l|l|l|l|l|l|}
\hline & $\begin{array}{l}\text { values } \\
\text { at } \mathbf{1}\end{array}$ & $\begin{array}{l}\text { Values } \\
\text { at 2 }\end{array}$ & $\begin{array}{l}\text { values } \\
\text { at } \mathbf{3}\end{array}$ & $\begin{array}{l}\text { values } \\
\text { at } \mathbf{4}\end{array}$ & $\begin{array}{l}\text { values } \\
\text { at 5 }\end{array}$ & $\begin{array}{l}\text { values } \\
\text { at 6 }\end{array}$ \\
\hline $\begin{array}{l}\text { Object } \\
\text { A }\end{array}$ & 18 & 20 & 30 & 21 & 34 & 32 \\
\hline $\begin{array}{l}\text { Object } \\
\text { B }\end{array}$ & 100 & 200 & 150 & 300 & 350 & 450 \\
\hline
\end{tabular}

The Chebyshev distance is 418 .

Pearson correlation distance

Table IV : Pearson correlation distance

\begin{tabular}{|c|c|c|c|c|c|c|}
\hline & $\begin{array}{c}\text { Values } \\
\text { at 1 }\end{array}$ & $\begin{array}{c}\text { Values } \\
\text { at 2 }\end{array}$ & $\begin{array}{c}\text { Values } \\
\text { at 3 }\end{array}$ & $\begin{array}{c}\text { Values } \\
\text { at 4 }\end{array}$ & $\begin{array}{c}\text { Values } \\
\text { at 5 }\end{array}$ & $\begin{array}{c}\text { Values } \\
\text { at 6 }\end{array}$ \\
\hline $\begin{array}{c}\text { Object } \\
\text { A }\end{array}$ & 18 & 20 & 30 & 21 & 34 & 32 \\
\hline $\begin{array}{c}\text { Object } \\
\text { B }\end{array}$ & 100 & 200 & 150 & 300 & 350 & 450 \\
\hline
\end{tabular}

$\mathrm{r}=0.6241$

Spearman correlation distance

$\mathrm{R}=0.7714$

Table V : Kendall correlation distance

Kendall tau Rank Correlation

Kendall tau $\quad 0.599999964237213$

2-sided p-value $\quad 0.13285493850708$

Score

9

Var(Score) $\quad 28.3333339691162$

Denominator $\quad 15.0000009536743$ 


\section{K-MEANS MAPREDUCE} ALGORITHM (KM-MR)

Input

$\mathrm{O}:\left\{\mathrm{o}_{1}, \mathrm{O}_{2}, \mathrm{O}_{3}, \ldots . . \mathrm{o}_{\mathrm{n}}\right\} ; / /$ number of objects to be clustered

$\mathrm{X}: \mathrm{X}$ number of clusters

$\mathrm{M}_{\mathrm{i}}$ : Maximum number of iterations

Table VI: Algorithm - Notations used

\begin{tabular}{|c|c|}
\hline Notation & Description \\
\hline i & Number of iterations \\
\hline IC & Starting centroid \\
\hline D & dataset \\
\hline PC & Nevious centroid values \\
\hline NC & Select data based on k value \\
\hline SELECT() & Function used for data file uploading \\
\hline INPUT() & Map Function \\
\hline job.mapper() & Reduce Funtion \\
\hline job.reducer() & Write centroid values to a file \\
\hline WRITE() & Read centroid values to a file \\
\hline READ() & Testing the updated centroid values \\
\hline UPDATE() & Inter cluster \\
\hline InC & Intra Cluster \\
\hline ItC & Euclidean Distance \\
\hline ECD & Nes \\
\hline
\end{tabular}

Output :

Desired output with number of clusters

K- Means - MR(values or data)

$\mathrm{i} \leftarrow 0$

For each datapoint $\mathrm{d} \in \mathrm{D}$ do

$\mathrm{IC} \leftarrow \operatorname{SELECT}(\mathrm{X}, \mathrm{d})$

INPUT(d)

WRITE(IC)

$\mathrm{PC} \leftarrow \mathrm{IC}$

while (true)

call to job.mapper()

call to job.reducer()

$\mathrm{NC}=\mathrm{READ}()$

If update $((\mathrm{NC}, \mathrm{PC})>0)$

$\mathrm{PC}=\mathrm{NC}$

else

update $\mathrm{NC}$ to result i++

result=READ ()

\section{MODIFIED K-MEANS CLUSTERING ALGORITHM (M - KM) \\ Map Phase Algorithm :}

Input :

M dimensional data objects $\left(\mathrm{m}_{1}, \mathrm{~m}_{2}, \mathrm{~m}_{3}, \ldots \ldots \mathrm{m}_{\mathrm{n}}\right)$ for each mapper

$\mathrm{X}:$ number of clusters

Read starting cluster centroids as $i_{1}, i_{2}, i_{3}, \ldots \ldots i_{k}$

Output:

output list $<\mathrm{a}, \mathrm{b}>$

list_new : new centroid list

set $\mathrm{k}=0$

list_new $=0$

for all $d \in D$

for all $i_{j} \in T$ do

bi $\sqsubset$ where bi represents centroid closest to the data object

$\mathrm{InC} \leftarrow \infty$

ItC $\leftarrow \infty$

For all $\mathrm{o}_{\mathrm{i}} \in \mathrm{O}$ do

$\mathrm{i} \leftarrow 0$

$1\left(\mathrm{o}_{\mathrm{i}}\right) \leftarrow$ Euclidean Distance $\left(\mathrm{o}_{\mathrm{i}}, \mathrm{o}_{\mathrm{j}}\right), \mathrm{j} \in\{1,2,3, \ldots . . \mathrm{k}\}$

$\mathrm{i} \leftarrow 0$

$\mathrm{b} \leftarrow 0$

repeat

for each $\mathrm{e}_{\mathrm{i}} \in \mathrm{E}$ do

$\operatorname{minDist} \leftarrow$ Euclidean Distance $\left(\mathrm{o}_{\mathrm{i}}, \mathrm{c}_{\mathrm{j}}\right), \mathrm{j} \in\{1,2,3, \ldots . . \mathrm{k}\}$

if (curr_centroid $=0$ or $1\left(\mathrm{o}_{\mathrm{i}}\right)<\operatorname{minDist}$ ) then

update InC

else

update ItC

$\mathrm{bi} \leftarrow \mathrm{bi}+1$

$\mathrm{i} \leftarrow \mathrm{i}+1$

create an output list $<\mathrm{a}, \mathrm{b}>$ with each object and the cluster centroid that it belongs to

repeat until convergence

\section{Reduce Phase Algorithm:}

Input :

Let $(\mathrm{a}, \mathrm{b}) \rightarrow$ key , value where $\mathrm{a}=1\left(\mathrm{o}_{\mathrm{i}}\right)$

value $=$ objects assigned centroids by mappers

$\mathrm{O}_{\mathrm{i}}$ represents mapper outputs

Output: 
list_new : new centroid list(NC)

list_new $=0$

$\mathrm{NC} \leftarrow \varnothing$

for all $\mathrm{x} \in \mathrm{O}_{1}$

centroid $\leftarrow$ x.key

data object $\leftarrow \mathrm{x}$.value

$\mathrm{NC} \leftarrow$ dataobject

for all $c_{i} \in M$ do

$\mathrm{NC} \leftarrow \varnothing$

sum_objects $\leftarrow \varnothing$

num_objects $\leftarrow \varnothing$

for all $\mathrm{o}_{\mathrm{i}} \in \mathrm{O}$ do

sum_objects $+=$ object

num_object++

$\mathrm{NC} \leftarrow$ (sum_objects/num_objects)

outputlist $\leftarrow \mathrm{NC}$ list

return $\mathrm{NC}$

Formula to calculate inter and intra clusters

$\operatorname{InC}=\frac{1}{2}\left(\sum_{i=1}^{O 1} \sum_{j=1}^{O 2}(A i-B j)^{2} / O 1 * O 2\right)$

$\mathrm{ItC}=\frac{1}{2}\left(\sum_{i, j=1}^{O 1+O 2}(A i-B j)^{2} /(01+O 2) *(01+02-1)\right)$

Where InC is inter cluster distance and $\mathrm{O} 1, \mathrm{O} 2$,.are data points in clusters 1,2 and so on.

$\mathrm{Ai}$ is ith data point in cluster 1 and jth data point in clusters $\mathrm{A}$ and $\mathrm{B}$.

\section{DATASETS}

The customer datasets that are freely available online. Apart from customer datasets, Iris datasets and US arrest datasets are taken for further processing. All the datasets can be downloaded for free from online that is mentioned in the references.

\section{RESULTS AND COMPARISON OF DATASETS FROM VARIOUS CALCULATIONS}

As mentioned in section III, K-Means algorithm is calculated in different Mathematical formats and the results are shown in the below figures.

K-Means algorithm works for all datasets, the graph is shown in figure 1. Comparison for the same is shown by taking various other Mathematical formulae that are applied to the same datasets. The graphs shown in figure 2, 3 and 4 are similar to the one showed in figure 1 .

Therefore our assumption for the above datasets is correct in other datasets that is proved mathematically.

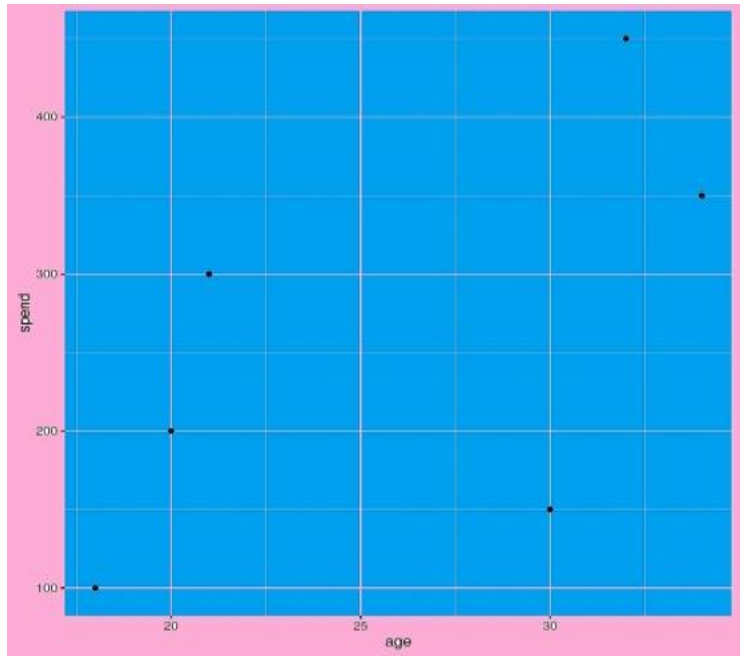

Figure 1: K-Means for Customer Datasets Overview

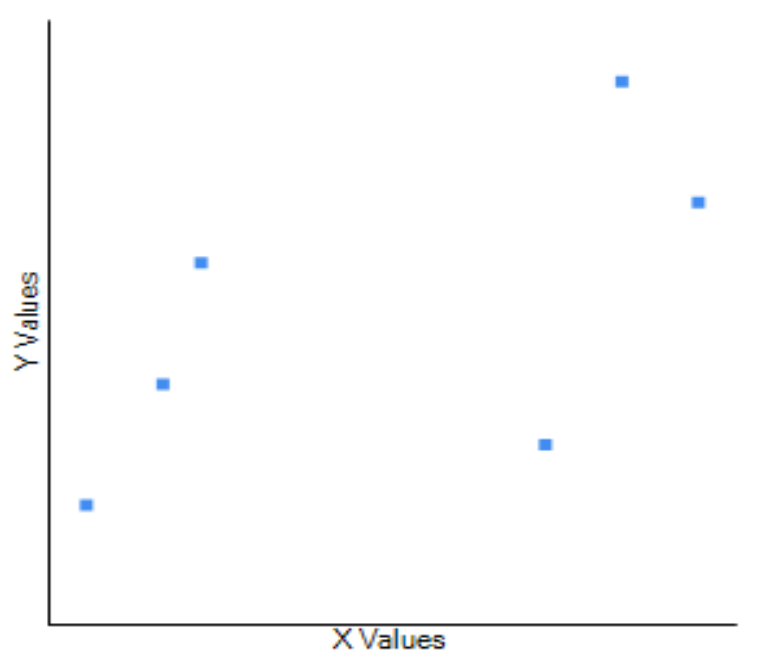

Figure 2: Pearson correlation distance

Scatterplot

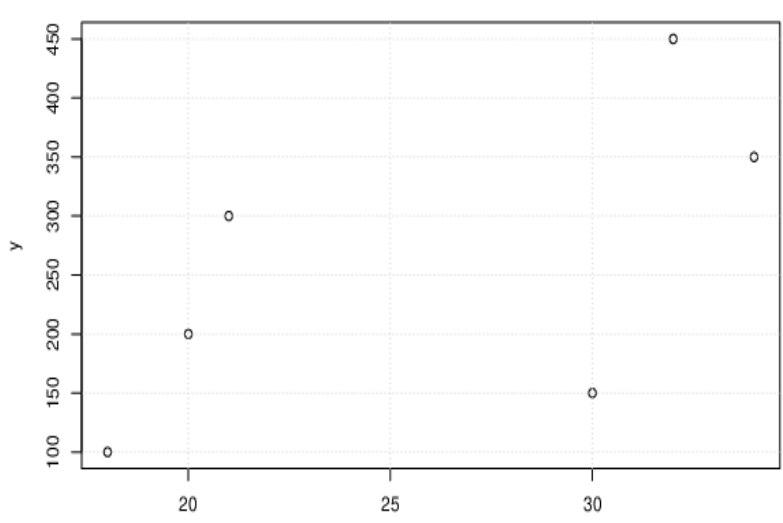

Figure 3 : Kendall correlation distance 


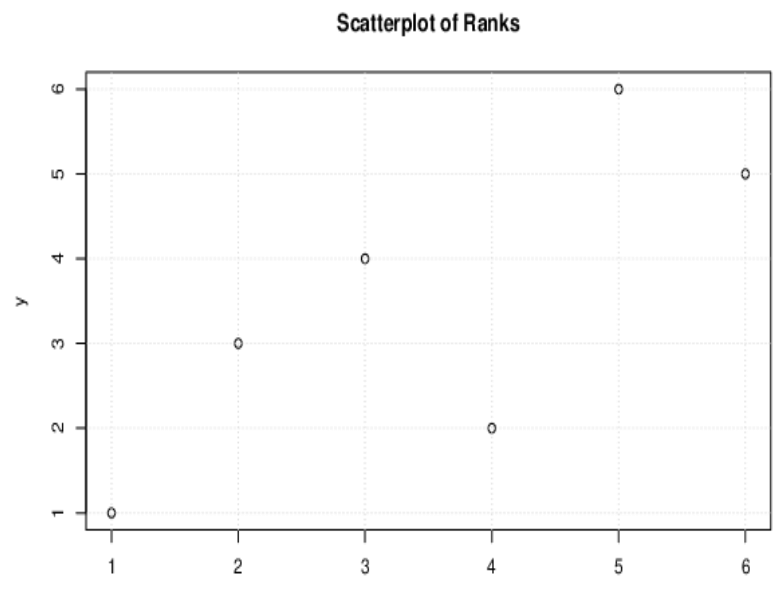

Figure 4 : Kendall correlation distance

\section{CONCLUSION}

Our approach for K-Means is applied for Customer datasets and proved to be correct. The same calculations can be applied for other datasets to verify the correctness of the approach. We are trying to apply the same for more complex and huge datasets and apply mathematical logic to prove our concept.

\section{REFERENCES}

[1] Jain, Anil K., M. Narasimha Murty, and Patrick J. Flynn. "Data clustering: a review." ACM computing surveys (CSUR) 31, no. 3 (1999): 264-323.

[2] Senthilnath, J., S. N. Omkar, and V. Mani. "Clustering using firefly algorithm: performance study." Swarm and Evolutionary Computation 1, no. 3 (2011): 164-171.
[3] Kanungo, Tapas, David M. Mount, Nathan S. Netanyahu, Christine D. Piatko, Ruth Silverman, and Angela Y. Wu. "An efficient k-means clustering algorithm: Analysis and implementation." Pattern Analysis and Machine Intelligence, IEEE Transactions on 24, no. 7 (2002): 881-892.

[4] Shyam Mohan J S, Shanmugapriya.P ,"Clustering of Huge Datasets using Machine Intelligence Techniques.’IJCA - Vol.181,No.18,September 2018.

[5] Robson L. F. Cordeiro et.al," Clustering Very Large Multi-dimensional Datasets with MapReduce." ACMKDD'11, August 21-24, 2011, San Diego, California, USA.

[6] Dongkuan Xu et.al," A Comprehensive Survey of Clustering Algorithms."Springer - Ann. Data. Sci. DOI 10.1007/s40745-015-0040-1.

[7] Max Bodoia ," MapReduce Algorithms for k-means Clustering."

[8] Nivranshu Hans et.al," Big Data Clustering Using Genetic Algorithm On Hadoop MapReduce." INTERNATIONAL JOURNAL OF SCIENTIFIC \& TECHNOLOGY RESEARCH VOLUME 4, ISSUE 04, APRIL 2015 ISSN 2277-8616.

[9] Sreedhar et al.,"Clustering large datasets using K means modified inter and intra clustering (KMI2C) in Hadoop", Journal Of Big Data , DOI 10.1186/s40537-017-0087-2, Springer 2017. 